We have considered the possibility of the spectrum being complex, but its shape seems to rule out such an explanation. The detector is such that $\gamma$-rays following emission of $\beta$-particles of a hypothetical softer group would have given rise to a readily observable discontinuity on our histograms, since the efficiency of detecting such $\gamma$-rays would be $\sim 25$ per cent. The life-time of the excited state of ${ }_{2} \mathrm{He}^{3}$ would have to be appreciably greater than the life-time of ${ }_{2} \mathrm{H}^{3}$ to escape detection.

In view of the above considerations, it is possible to justify the application of neutrino theory to the upper end of the spectrum in the usual manner. However, we have shown in the inset figure two curves calculated for neutrino masses $\mu=m / 125$ and $m / 300$, and comparison of these with experiment seems to indicate that $\mu$ is less than $m / 300$.

Since there is no evidence of $\gamma$-radiation following the $\beta$-emission, we can write the mass equation (atomic) as

$$
{ }_{1} \mathrm{H}^{3}-{ }_{2} \mathrm{He}^{3}=0.000018 \text {. }
$$

It is a pleasure to thank Prof. P. I. Dee for his valuable advice throughout the work. We are grateful to Mr. B. Touschek for elucidating various theoretical points.

$$
\begin{aligned}
& \text { S. C. Curran } \\
& \text { J. Angus } \\
& \text { A. L. Cockcroft }
\end{aligned}
$$

Department of Natural Philosophy,

University of Glasgow. May 21 .

${ }^{1}$ Konopinski, E. J., Phys. Rev., 72, 518 (1947).

${ }^{2}$ Novick, A., Phys. Rev., 72, 972 (1947). Goldblatt, M., et al., Phys. Rev., 72, 973 (1947).

${ }^{3}$ Watts, R. J., and Williams, D., Phys. Rev., 70, 640 (1946).

"Comptor, A. H., and Allison, S. K., "X-Rays" (Macmillan, 1936).

\section{Velocity of Photons}

I AM seeking an answer-or a reference to an answer-to an apparent paradox arising out of the theory that light is transmitted in the form of photons, which may be illustrated by the following concrete example.

Atoms of sodium on Sirius, vibrating so as to give out a definite spectral line, send out photons each of which contains the amount of energy $(h \nu)$ corresponding to such vibration. These photons, therefore, are identical in all respects, since their energy content is dictated solely by local conditions and clearly cannot be influenced by what may be their ultimate targets, if any. Two such photons are directed towards the solar system, and one arrives at the earth which is approaching Sirius at 30 miles per second, and the other on Mars which happens to be receding from Sirius with the same velocity. What physical factor causes these photons to give different optical effects since $(a)$ their internal energies are identical, $(b)$ their speeds are also identical, since they arrive at their targets with the velocity (absolute and relative) of light, which is independent of the motions of source and observer?

I have searched in vain for any explanation of the Doppler effect which recognizes (a) that light moves in the form of photons, and $(b)$ that the velocity of the photons is independent of that of their source.

Personally, I can soe no answer to this query unless the theory as to the travel of light in the form of photons is profoundly modified.

\section{H. Fletcher Moulton}

4 King's Bench Walk, Temple, E.C.4.

\section{Latitude Effect and Meson Decay}

IT has been pointed out recently ${ }^{1}$ that the height of the atmospheric layer (100 mb.) in which most mesons are supposed to be produced decreases from the equator towards the poles. The decay of the mesons causes a corresponding increase in cosmic ray intensity.

A first calculation of this effect based on a decay constant $^{2,3} L=5 \mathrm{~km}$. seemed to indicate that the greater part of, if not all, the latitude effect might be explained thus. A more precise estimation of the 'atmospheric latitude of effect' as distinguished from the geomagnetic can be obtained as follows. The altitude of the $100-\mathrm{mb}$. level varies not only with geographic latitude but also with the seasons. It is assumed that the seasonal effect of cosmic rays as observed in latitudes where the geomagnetic effect is small, if not nil, is entirely due to these variations. This is called the 'atmospheric seasonal effect'. Further, the 'atmospheric latitude effect' is defined as the variations in cosmic ray intensity caused by the differences at different geographic latitudes in the yearly averages of the heights at which mesons are produced; all measurements having been reduced before to standard atmospheric pressure. The ratio of the two effects can be obtained by comparing the seasonal variation of the meson-producing layer with its average latitudinal variation between the equator and the latitude under consideration. The influence of a differing energy spectrum is neglected.

Measurements with shielded ionization chambers at sea-level ${ }^{4,5}$ and tables and graphs of the altitude of the $100-\mathrm{mb}$. level $\mathbf{l}^{6,7}$ supply all the data required. These are tabulated in columns 1, 2, 3, 4.

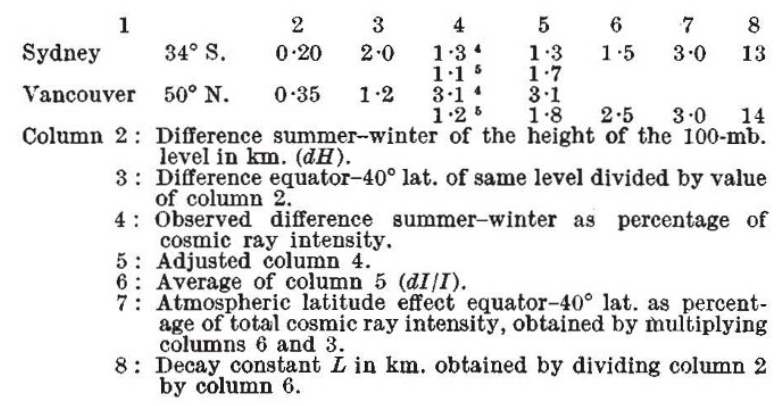

The values for some of the seasonal variations of cosmic rays have to be adjusted for the difference in the period over which they and the stratosphere data were averaged (column 5). The decay constant $L$ was calculated from the relation $d I / I=-d H / L$, in which $I$ is the cosmic ray intensity and $H$ the height. Taking into consideration the wide differences possible in the measurements of the heights at times as well as localities far removed from the cosmic ray measurements, it is satisfactory to find that the results obtained for the decay constant lie within the range $e^{8, \theta}$ of $9-19 \mathrm{~km}$. of the most reliable determ. inations. In conclusion, it can be stated that the atmospheric latitude effect is about three per cent of the total intensity, that is, one third of the total latitude effect between the equator and the knee at sea-level. This reduction of the geomagnetic latitude effect, if confirmed, will require a revision of the energy spectrum of the primary cosmic rays.

The east-west effect, the nearly constant geomagnetic latitude of the knee, demonstrate also that a great part of the latitude effect is of magnetic origin. 